\title{
Distribution functions for a two-dimensional non-interacting quantum electron gas in an external magnetic field
}

\author{
F. LADO* \\ Department of Physics, North Carolina State University, Raleigh, North Carolina \\ 27695-8202, USA
}

(Received 3 October 2002; revised version accepted 28 November 2002)

\begin{abstract}
The exact $n$-body distribution functions are calculated for a two-dimensional, non-interacting quantum electron gas in an external magnetic field for any temperature and density. At low tempertures and filled lowest Landau level (LLL), these functions are identical to the exact distribution functions obtained by Jancovici [1981, Phys. Rev. Lett., 46, 386] for the classical two-dimensional one-component plasma (2DOCP) at the special plasma parameter $\Gamma=2$, thus establishing that the 2DOCP provides an exact classical Boltzmann factor which describes the ideal LLL quantum state associated with the integral quantum Hall effect.
\end{abstract}

\section{Introduction}

It was not so long ago that physical models in fewer than three dimensions were primarily of interest to theorists seeking greater tractability for the mathematics used to describe the systems in three dimensions. Experiments carried out in recent decades, however, have demonstrated that a two-dimensional world is not an arcane corner of theoretical physics but a real presence with measurable and often fascinating properties. In the process, many once esoteric models have gained fresh immediacy. In particular, a system of non-interacting electrons in a plane subject to an external magnetic field, first studied by Landau [1] in 1930 to account for diamagnetism, became the basis for understanding the integral quantum Hall effect [2], discovered by von Klitzing et al. [3] 50 years later. The discovery of the fractional quantum Hall effect [4] not long after called for a different explanation and an important contribution was made by Laughlin [5], who invoked the classical two-dimensional one-component plasma $[6,7]$ in constructing his solution.

In this work, on the occasion of Dominique Levesque's 65th birthday, we report a surprising connection between the same two-dimensional onecomponent plasma (2DOCP) and the integral quantum Hall effect, as modelled by the simplest system, Landau's non-interacting electrons in a plane, subject to an external magnetic field perpendicular to the plane. The connection is made after calculating the exact $n$-body distribution functions of this system and realizing that, in a certain limit, they are identical to the exact

*e-mail: fred_lado@ncsu.edu distribution functions of the 2DOCP found by Jancovici [8] for plasma parameter $\Gamma=2$.

\section{General $n$-particle distribution functions}

\subsection{Preliminaries}

We consider $N$ electrons at temperature $T$ in area $A$. A uniform magnetic field $B_{0}$ is applied perpendicular to the plane of $A$. The canonical partition function $Q_{N}$ for this system can be written

$$
Q_{N}=\frac{1}{N ! \Lambda^{2 N}} \int W_{N}\left(\mathbf{r}^{N}\right) \mathrm{d} \mathbf{r}_{1} \cdots \mathrm{d} \mathbf{r}_{N}
$$

where $W_{N}\left(\mathbf{r}^{N}\right)$ is the (antisymmetrized) Slater sum,

$$
\begin{aligned}
W_{N}\left(\mathbf{r}^{N}\right)= & \Lambda^{2 N} \sum_{P}(-1)^{|P|} \sum_{k} \Psi_{k}^{*}\left(P \mathbf{r}^{N}\right) \\
& \times \exp (-\beta \mathcal{H}) \Psi_{k}\left(\mathbf{r}^{N}\right),
\end{aligned}
$$

which plays the role of Boltzmann factor. The conventional introduction of the thermal de Broglie wavelength $\Lambda=\left(h^{2} / 2 \pi m_{\mathrm{e}} k_{\mathrm{B}} T\right)^{1 / 2}$ in equation (1) serves to make the Slater sum dimensionless but has no effect on the results reported below. Here the $\Psi_{k}$ are a complete set of $N$ particle states distinguished by index $k$ representing $2 N$ quantum numbers; the sum over permutations $P$, with even or odd parity $|P|$ and antisymmetric weighting, accounts for the Fermi-Dirac statistics of the indistinguishable electrons. The Hamiltonian $\mathcal{H}$ for noninteracting electrons in an external magnetic field is

$$
\mathcal{H}_{N}=\frac{1}{2 m_{\mathrm{e}}} \sum_{j=1}^{N}\left[-\mathrm{i} \hbar \nabla_{j}+e \mathbf{A}\left(\mathbf{r}_{j}\right)\right]^{2},
$$


where $m_{\mathrm{e}}$ is the electron mass, $-e$ is its charge and $\mathbf{A}(\mathbf{r})$ is a vector potential which yields a uniform magnetic field $\mathbf{B}_{0}$ (which we shall take to define the $z$ direction),

$$
\mathbf{B}_{0}=B_{0} \hat{\mathbf{k}}=\nabla \times \mathbf{A}(\mathbf{r}) .
$$

As usual, $\beta=1 / k_{\mathrm{B}} T$ is the inverse temperature, with $k_{\mathrm{B}}$ being Boltzmann's constant. The system is taken to be fully spin-polarized, so that spin degrees of freedom may be ignored.

\subsection{The Slater sum}

Since interactions between electrons are being omitted, the Schrödinger equation for the energy eigenstates is readily solved and the sum over states in equation (2) can be carried out. The vector potential in the Landau gauge is $\mathbf{A}=-B_{0} y \hat{\boldsymbol{i}}$, so the one-electron Hamiltonian becomes

$$
\mathcal{H}_{1}=\frac{1}{2 m_{\mathrm{e}}}\left[\left(\mathrm{i} \hbar \frac{\partial}{\partial x}+e B_{0} y\right)^{2}+\left(\mathrm{i} \hbar \frac{\partial}{\partial y}\right)^{2}\right] .
$$

The Schrödinger equation $\mathcal{H}_{1} \psi=\epsilon \psi$ is then solved by [2]

$$
\psi_{\kappa n}(x, y)=\frac{A_{n}}{L_{x}^{1 / 2}} \exp \left[\mathrm{i} \kappa x-\frac{\left(y-\kappa l_{0}^{2}\right)^{2}}{2 l_{0}^{2}}\right] H_{n}\left(\frac{y-\kappa l_{0}^{2}}{l_{0}}\right),
$$

with $\kappa=2 \pi j / L_{x}$ for $j=0, \pm 1, \pm 2, \ldots, l_{0}=\left(\hbar / e B_{0}\right)^{1 / 2}$, and $A_{n}=\left(\pi l_{0}^{2}\right)^{-1 / 4}\left(2^{n} n !\right)^{-1 / 2}$. Further, $H_{n}(x)$ is the Hermite polynomial of order $n$ and $L_{x}$ is the edge length of the rectangular area $A$ in the $x$ direction. The corresponding energy eigenvalues are $\epsilon_{n}=(2 n+1) \mu_{\mathrm{B}} B_{0}$, $n=0,1,2, \ldots$, where $\mu_{\mathrm{B}}=e \hbar / 2 m_{\mathrm{e}}$ is the Bohr magneton.

We seek to determine the one-body Slater sum

$$
\begin{aligned}
W_{1}(x, y)= & \Lambda^{2} \sum_{\kappa, n} \exp \left(-\beta \epsilon_{n}\right) \psi_{\kappa n}^{*}(P x, P y) \psi_{\kappa n}(x, y) \\
= & \frac{\Lambda^{2}}{\left(\pi l_{0}^{2}\right)^{1 / 2} L_{x}} \sum_{\kappa} \exp [\mathrm{i} \kappa(x-P x)] \\
& \times \exp \left[-\left(P y-\kappa l_{0}^{2}\right)^{2} / 2 l_{0}^{2}\right] \\
& \times \exp \left[-\left(y-\kappa l_{0}^{2}\right)^{2} / 2 l_{0}^{2}\right] \\
& \times \sum_{n} \frac{\exp \left[-(2 n+1) \beta \mu_{\mathrm{B}} B_{0}\right]}{2^{n} n !} \\
& \times H_{n}\left(\frac{P y-\kappa l_{0}^{2}}{l_{0}}\right) H_{n}\left(\frac{y-\kappa l_{0}^{2}}{l_{0}}\right) .
\end{aligned}
$$

We note first that [9]

$$
\begin{aligned}
\sum_{n=0}^{\infty} \frac{\exp (-n \alpha)}{2^{n} n !} H_{n}(x) H_{n}(y) \\
\quad=\left(\frac{\exp (\alpha)}{2 \sinh \alpha}\right)^{1 / 2} \exp \left[\frac{-\left(x^{2}+y^{2}\right) \exp (-\alpha)+2 x y}{2 \sinh \alpha}\right] .
\end{aligned}
$$

Using this in equation (7) with $\alpha=2 \beta \mu_{\mathrm{B}} B_{0}$ and simplifying, we arrive at

$$
\begin{aligned}
W_{1}(x, y)= & {\left[\Lambda^{2} /\left(2 \pi l_{0}^{2} \sinh \alpha\right)^{1 / 2} L_{x}\right] \sum_{\kappa} \exp [\mathrm{i} \kappa(x-P x)} \\
& -(y-P y)^{2} / 2 l_{0}^{2} \tanh \alpha \\
& \left.-\tanh (\alpha / 2)\left(y-\kappa l_{0}^{2}\right)\left(P y-\kappa l_{0}^{2}\right) / l_{0}^{2}\right]
\end{aligned}
$$

The second sum, over $\kappa$, is effected as an integral. For large $L_{x}, L_{x}^{-1} \sum_{\kappa} \rightarrow(2 \pi)^{-1} \int \mathrm{d} \kappa$ and we get

$$
\begin{aligned}
& \frac{1}{2 \pi} \int_{-\infty}^{\infty} \mathrm{d} \kappa \exp [\mathrm{i} \kappa(x-P x) \\
&\left.-\tanh (\alpha / 2)\left(y-\kappa l_{0}^{2}\right)\left(P y-\kappa l_{0}^{2}\right) / l_{0}^{2}\right] \\
&=\left[4 \pi l_{0}^{2} \tanh (\alpha / 2)\right]^{-1 / 2} \\
& \quad \times \exp \left[-(x-P x)^{2} / 4 l_{0}^{2} \tanh (\alpha / 2)\right. \\
&+\tanh (\alpha / 2)(y-P y)^{2} / 4 l_{0}^{2} \\
&\left.+\mathrm{i}(x-P x)(y+P y) / 2 l_{0}^{2}\right],
\end{aligned}
$$

and so finally

$$
\begin{aligned}
W_{1}(x, y)= & {\left[\Lambda^{2} / 4 \pi l_{0}^{2} \sinh (\alpha / 2)\right] } \\
& \times \exp \left[-(\mathbf{r}-P \mathbf{r})^{2} / 4 l_{0}^{2} \tanh (\alpha / 2)\right. \\
& \left.+\mathrm{i}(x-P x)(y+P y) / 2 l_{0}^{2}\right],
\end{aligned}
$$

after some further simplification.

The final result for the $N$-body Slater sum is

$$
\begin{aligned}
W_{N}\left(\mathbf{r}^{N}\right)= & \left(\Lambda^{2} / 4 \pi l_{0}^{2} \sinh \beta \mu_{\mathrm{B}} B_{0}\right)^{N} \sum_{P}(-1)^{|P|} \\
& \times \exp \left\{\sum _ { j = 1 } ^ { N } \left[-\left(\mathbf{r}_{j}-P \mathbf{r}_{j}\right)^{2} / 4 l_{0}^{2} \tanh \beta \mu_{\mathrm{B}} B_{0}\right.\right. \\
& \left.\left.+\mathrm{i}\left(x_{j} P y_{j}-y_{j} P x_{j}\right) / 2 l_{0}^{2}\right]\right\},
\end{aligned}
$$

where $\mathbf{r}_{j}$ locates electron $j$ in the $x y$ plane, $\mu_{\mathrm{B}}$ is the Bohr magneton and $l_{0}=\left(\hbar / e B_{0}\right)^{1 / 2}$ is the characteristic magnetic length. Letting the external field $B_{0}$ vanish recovers the Slater sum of ideal fermions, 


$$
\left.W_{N}\left(\mathbf{r}^{N}\right)\right\rfloor_{B_{0}=0}=\sum_{P}(-1)^{|P|} \exp \left[-\frac{\pi}{\Lambda^{2}} \sum_{j=1}^{N}\left(\mathbf{r}_{j}-P \mathbf{r}_{j}\right)^{2}\right] \text {. }
$$

\subsection{The distribution functions}

With the Slater sum in hand, we now seek to determine the $n$-body distribution function

$$
\rho_{N}^{(n)}\left(\mathbf{r}_{1}, \ldots, \mathbf{r}_{n}\right)=\frac{1}{(N-n) ! \Lambda^{2 N} Q_{N}} \int W_{N}\left(\mathbf{r}^{N}\right) \mathrm{d} \mathbf{r}_{n+1} \ldots \mathrm{d} \mathbf{r}_{N}
$$

for any $n$. One notes that each permutation $P$ in equation (12) produces a disjoint set of ring-type integrands, with each particle appearing in one and only one ring. Particle positions $1,2, \ldots, n$ are not integrated out and will be called root points. The remaining $N-n$ particle positions are variables of integration, to be called field points. The bond linking adjacent particles $i$ and $j$ in a ring is

$$
\begin{aligned}
f\left(\mathbf{r}_{i}, \mathbf{r}_{j}\right)= & \left(4 \pi l_{0}^{2} \sinh \beta \mu_{\mathrm{B}} B_{0}\right)^{-1} \\
& \times \exp \left[-\left(\mathbf{r}_{i}-\mathbf{r}_{j}\right)^{2} / 4 l_{0}^{2} \tanh \beta \mu_{\mathrm{B}} B_{0}\right. \\
& \left.+\mathrm{i}\left(x_{i} y_{j}-y_{i} x_{j}\right) / 2 l_{0}^{2}\right] .
\end{aligned}
$$

Let $I_{k}\left(\mathbf{r}_{i}, \mathbf{r}_{j}\right)$ be a chain of links beginning at root point $\mathbf{r}_{i}$ and ending at root point $\mathbf{r}_{j}$ with $k$ field points in between; i.e.

$$
I_{k}\left(\mathbf{r}_{i}, \mathbf{r}_{j}\right)=\int f\left(\mathbf{r}_{i}, \mathbf{r}_{n_{1}}\right) f\left(\mathbf{r}_{n_{1}}, \mathbf{r}_{n_{2}}\right) \cdots f\left(\mathbf{r}_{n_{k}}, \mathbf{r}_{j}\right) \mathrm{d} \mathbf{r}_{n_{1}} \cdots \mathrm{d} \mathbf{r}_{n_{k}} .
$$

The canonical distribution function $\rho_{N}^{(n)}\left(\mathbf{r}_{1}, \ldots, \mathbf{r}_{n}\right)$ then becomes [10]

$$
\begin{aligned}
\rho_{N}^{(n)}\left(\mathbf{r}_{1}, \ldots, \mathbf{r}_{n}\right)= & \frac{1}{(N-n) ! Q_{N}} \sum_{P}(-1)^{|P|} \\
& \times \sum_{m=0}^{N-n}(-1)^{m} \frac{(N-n) !}{(N-n-m) !} \\
& \times \sum_{k_{1}, k_{2}, \ldots, k_{n}} \delta\left(m, \sum_{j} k_{j}\right) I_{k_{1}}\left(\mathbf{r}_{1}, P \mathbf{r}_{1}\right) \\
& \times I_{k_{2}}\left(\mathbf{r}_{2}, P \mathbf{r}_{2}\right) \cdots I_{k_{n}}\left(\mathbf{r}_{n}, P \mathbf{r}_{n}\right) \\
& \times(N-n-m) ! Q_{N-n-m} .
\end{aligned}
$$

Now the permutations $P$ are among the $n$ root points only, while $m$ is the total number of field points in the rooted rings. The sums over number of field points $k_{j}$ in individual chains are collectively constrained by the requirement $\sum_{j=1}^{n} k_{j}=m$, expressed by the Kronecker delta $\delta(i, j)$ in the summand. This constraint can be removed, and the individual sums over the $k_{j}$ factored, by transforming these expressions from particle number $N$ to chemical potential $\mu$ as an independent variable using the grand canonical formalism. We have then

$$
\Xi=\sum_{N=0}^{\infty} \exp (\beta \mu N) Q_{N}
$$

for the partition function and

$$
\rho^{(n)}\left(\mathbf{r}_{1}, \ldots, \mathbf{r}_{n}\right)=\frac{1}{\Xi} \sum_{N=n}^{\infty} \exp (\beta \mu N) Q_{N} \rho_{N}^{(n)}\left(\mathbf{r}_{1}, \ldots, \mathbf{r}_{n}\right)
$$

for the $n$-body distribution function. Using equation (17) in the latter we now get simply

$$
\begin{aligned}
\rho^{(n)}\left(\mathbf{r}_{1}, \ldots, \mathbf{r}_{n}\right)= & \sum_{P}(-1)^{|P|} \prod_{j=1}^{n} \\
& \times\left[\sum_{k=0}^{\infty}(-1)^{k} \exp [(k+1) \beta \mu] I_{k}\left(\mathbf{r}_{j}, P \mathbf{r}_{j}\right)\right] .
\end{aligned}
$$

Starting from $I_{0}\left(\mathbf{r}_{i}, \mathbf{r}_{j}\right)=f\left(\mathbf{r}_{i}, \mathbf{r}_{j}\right)$, one finds by induction that

$$
\begin{aligned}
I_{k}\left(\mathbf{r}_{i}, \mathbf{r}_{j}\right)= & {\left[4 \pi l_{0}^{2} \sinh \left((k+1) \beta \mu_{\mathrm{B}} B_{0}\right)\right]^{-1} } \\
& \times \exp \left[-\left(\mathbf{r}_{i}-\mathbf{r}_{j}\right)^{2} / 4 l_{0}^{2} \tanh \left((k+1) \beta \mu_{\mathrm{B}} B_{0}\right)\right. \\
& \left.+\mathrm{i}\left(x_{i} y_{j}-y_{i} x_{j}\right) / 2 l_{0}^{2}\right],
\end{aligned}
$$

so that finally

$$
\begin{aligned}
\rho^{(n)}\left(\mathbf{r}_{1}, \ldots, \mathbf{r}_{n}\right)= & \sum_{P}(-1)^{|P|} \prod_{j=1}^{n}\left\{\eta\left(\left|\mathbf{r}_{j}-P \mathbf{r}_{j}\right|\right)\right. \\
& \left.\times \exp \left[\mathrm{i}\left(x_{j} P y_{j}-y_{j} P x_{j}\right) / 2 l_{0}^{2}\right]\right\},
\end{aligned}
$$

with

$$
\begin{aligned}
\eta(r)= & \frac{1}{4 \pi l_{0}^{2}} \sum_{j=1}^{\infty} \frac{(-1)^{j-1} \exp (j \beta \mu)}{\sinh \left(j \beta \mu_{\mathrm{B}} B_{0}\right)} \\
& \times \exp \left[-r^{2} / 4 l_{0}^{2} \tanh \left(j \beta \mu_{\mathrm{B}} B_{0}\right)\right] .
\end{aligned}
$$

The first of these distribution functions,

$$
\rho^{(1)}\left(\mathbf{r}_{1}\right)=\eta(0),
$$

is just the uniform density $\rho=\bar{N} / A$ expressed in terms of the chemical potential $\mu$,

$$
\rho=\frac{1}{4 \pi l_{0}^{2}} \sum_{j=1}^{\infty} \frac{(-1)^{j-1} \exp (j \beta \mu)}{\sinh \left(j \beta \mu_{\mathrm{B}} B_{0}\right)} .
$$

This result is more familiarly obtained directly from the partition function $\Xi$, where 


$$
\ln \Xi=\frac{A}{4 \pi l_{0}^{2}} \sum_{j=1}^{\infty} \frac{(-1)^{j-1} \exp (j \beta \mu)}{j \sinh \left(j \beta \mu_{\mathrm{B}} B_{0}\right)},
$$

using the thermodynamic relation $\bar{N}=k_{\mathrm{B}} T \partial \ln \Xi / \partial \mu$.

The dimensionless forms

$$
g^{(n)}\left(\mathbf{r}_{1}, \ldots, \mathbf{r}_{n}\right) \equiv \rho^{(n)}\left(\mathbf{r}_{1}, \ldots, \mathbf{r}_{n}\right) / \rho^{n}
$$

are preferable in writing out higher distribution functions. Explicitly, we have for the next three,

$$
\begin{aligned}
g^{(2)}\left(\mathbf{r}_{1}, \mathbf{r}_{2}\right)= & 1-D^{2}\left(r_{12}\right), \\
g^{(3)}\left(\mathbf{r}_{1}, \mathbf{r}_{2}, \mathbf{r}_{3}\right)= & 1-\left[D^{2}\left(r_{12}\right)+D^{2}\left(r_{13}\right)+D^{2}\left(r_{23}\right)\right] \\
& +2 D\left(r_{12}\right) D\left(r_{23}\right) D\left(r_{31}\right) \\
& \times \cos \left(\left|\mathbf{r}_{12} \times \mathbf{r}_{13}\right| / 2 l_{0}^{2}\right) \\
& \quad g^{(4)}\left(\mathbf{r}_{1}, \mathbf{r}_{2}, \mathbf{r}_{3}, \mathbf{r}_{4}\right) \\
= & 1-\left[D^{2}\left(r_{12}\right)+D^{2}\left(r_{13}\right)+D^{2}\left(r_{14}\right)\right. \\
+ & \left.D^{2}\left(r_{23}\right)+D^{2}\left(r_{24}\right)+D^{2}\left(r_{34}\right)\right] \\
+ & {\left[D^{2}\left(r_{12}\right) D^{2}\left(r_{34}\right)+D^{2}\left(r_{13}\right) D^{2}\left(r_{24}\right)+D^{2}\left(r_{14}\right) D^{2}\left(r_{23}\right)\right] } \\
+ & 2\left[D\left(r_{12}\right) D\left(r_{23}\right) D\left(r_{31}\right) \cos \left(\left|\mathbf{r}_{12} \times \mathbf{r}_{13}\right| / 2 l_{0}^{2}\right)\right. \\
+ & D\left(r_{13}\right) D\left(r_{34}\right) D\left(r_{41}\right) \cos \left(\left|\mathbf{r}_{13} \times \mathbf{r}_{14}\right| / 2 l_{0}^{2}\right) \\
+ & D\left(r_{12}\right) D\left(r_{24}\right) D\left(r_{41}\right) \cos \left(\left|\mathbf{r}_{12} \times \mathbf{r}_{14}\right| / 2 l_{0}^{2}\right) \\
+ & \left.D\left(r_{23}\right) D\left(r_{34}\right) D\left(r_{42}\right) \cos \left(\left|\mathbf{r}_{23} \times \mathbf{r}_{24}\right| / 2 l_{0}^{2}\right)\right] \\
& -2\left[D\left(r_{12}\right) D\left(r_{23}\right) D\left(r_{34}\right) D\left(r_{41}\right) \cos \left(\left|\mathbf{r}_{13} \times \mathbf{r}_{24}\right| / 2 l_{0}^{2}\right)\right. \\
& +D\left(r_{12}\right) D\left(r_{24}\right) D\left(r_{43}\right) D\left(r_{31}\right) \cos \left(\left|\mathbf{r}_{14} \times \mathbf{r}_{23}\right| / 2 l_{0}^{2}\right) \\
+ & \left.D\left(r_{13}\right) D\left(r_{32}\right) D\left(r_{24}\right) D\left(r_{41}\right) \cos \left(\left|\mathbf{r}_{12} \times \mathbf{r}_{34}\right| / 2 l_{0}^{2}\right)\right]
\end{aligned}
$$

where $D(r)=\eta(r) / \eta(0)$. The three-body distribution function is the first to show correlations due to phase.

A key result here, $\eta(r)$ in equation (23), can be rewritten in a form that more directly displays the familiar Fermi degeneracy at low temperatures. We use the generating function of Laguerre polynomials,

$$
\frac{\exp [-x z /(1-z)]}{1-z}=\sum_{k=0}^{\infty} L_{k}(x) z^{k}
$$

with $x=r^{2} / 2 l_{0}^{2}$ and $z=\exp \left(-2 j \beta \mu_{\mathrm{B}} B_{0}\right)$ in equation (23) and then sum over $j$ to get

$$
\begin{aligned}
\eta(r)= & \frac{\exp \left(-r^{2} / 4 l_{0}^{2}\right)}{2 \pi l_{0}^{2}} \\
& \times \sum_{k=0}^{\infty} \frac{L_{k}\left(r^{2} / 2 l_{0}^{2}\right)}{1+\exp \left\{-\beta\left[\mu-(2 k+1) \mu_{\mathrm{B}} B_{0}\right]\right\}},
\end{aligned}
$$

where now the summation is over Landau levels, of energy $\epsilon_{k}=(2 k+1) \mu_{\mathrm{B}} B_{0}$. For completeness, we note that the expansion $1 / \sinh x=2 \sum_{k=0}^{\infty} \exp [-(2 k+1) x]$ used similarly in equations (25) and (26) gives

$$
\begin{aligned}
\rho & =\frac{1}{2 \pi l_{0}^{2}} \sum_{k=0}^{\infty} \frac{1}{1+\exp \left(-\beta\left[\mu-(2 k+1) \mu_{\mathrm{B}} B_{0}\right]\right)}, \\
\ln \Xi & =\frac{A}{2 \pi l_{0}^{2}} \sum_{k=0}^{\infty} \ln \left\{1+\exp \left(\beta\left[\mu-(2 k+1) \mu_{\mathrm{B}} B_{0}\right]\right)\right\} .
\end{aligned}
$$

For low temperatures and large magnetic fields, i.e. for $\beta \mu_{\mathrm{B}} B_{0} \gg 1$, the summand in equation (33) acts like a discrete unit step function, so that for (integer) $n$ filled Landau levels one has $2 \pi \rho l_{0}^{2}=n$; similarly, using equation (32), the pair distribution function $g^{(2)}\left(r_{12}\right)=$ $g^{(2)}\left(\mathbf{r}_{1}, \mathbf{r}_{2}\right)$ simplifies in this regime to

$$
\left.g^{(2)}(r)\right\rfloor_{T=0}=1-\exp \left(-r^{2} / 2 l_{0}^{2}\right)\left[\frac{1}{n} \sum_{k=0}^{n-1} L_{k}\left(r^{2} / 2 l_{0}^{2}\right)\right]^{2},
$$

a result that has been obtained by Ciftja and Fantoni [11] and by Kamilla et al. [12].

\subsection{A remarkable connection}

We close this work by noting a remarkable fact. For physical conditions such that this non-interacting quantum electron system just fills the lowest Landau level, i.e. for $\beta \mu_{\mathrm{B}} B_{0} \gg 1$ and $2 \pi \rho l_{0}^{2}=1$, so that $D(r)=\exp \left(-\pi \rho r^{2} / 2\right)$, the exact distribution functions $g^{(n)}\left(\mathbf{r}_{1}, \ldots, \mathbf{r}_{n}\right)$ displayed above are identical to the exact $g^{(n)}\left(\mathbf{r}_{1}, \ldots, \mathbf{r}_{n}\right)$ of a classical two-dimensional one-component plasma (2DOCP) obtained by Jancovici [8] for the special plasma parameter value $\Gamma=2$. Working backwards, we may then conclude that the quantum state for the filled lowest Landau level is precisely described by an effective classical pair potential $\phi(r)=-2 \ln \left(r / l_{0}\right)$. Adapting to the present notation the total potential $V$ quoted by Jancovici [8] for 2DOCP particles confined to a disc of radius $R$, we get a system potential

$\Phi_{N}^{(1)}\left(\mathbf{r}^{N}\right)=-2 \sum_{i<j} \ln \left(\frac{r_{i j}}{l_{0}}\right)+\frac{1}{2} \sum_{i}\left(\frac{r_{i}}{l_{0}}\right)^{2}+N^{2}\left(\ln \frac{R}{l_{0}}-\frac{3}{4}\right)$,

or, equivalently, a Boltzmann factor $W=\exp (-\Phi)$ given by

$$
W_{N}^{(1)}\left(\mathbf{r}^{N}\right)=\prod_{i<j}\left(\frac{r_{i j}}{l_{0}}\right)^{2} \exp \left[-\frac{1}{2} \sum_{i}\left(\frac{r_{i}}{l_{0}}\right)^{2}\right],
$$

neglecting multiplicative constants, where $r_{i}$ is the radial distance from the disc centre. The non-interacting electron gas in two dimensions is the basis for 
understanding the integral quantum Hall effect [2]; we find that for the incompressible state at low temperatures with filling factor $\nu=1$, this system is exactly described by the effective Boltzmann factor written above. Further, the wave function constructed by Laughlin [5] to explain the fractional quantum Hall effect for filling factor $\nu=1 / m$, with $m$ an odd integer, produces just this same Boltzmann factor,

$$
W_{N}^{(1 / m)}\left(\mathbf{r}^{N}\right)=\prod_{i<j}\left(\frac{r_{i j}}{l_{0}}\right)^{2 m} \exp \left[-\frac{1}{2} \sum_{i}\left(\frac{r_{i}}{l_{0}}\right)^{2}\right],
$$

for $m=1$. One concludes that, at least for the filled lowest Landau level, $\nu=1 / m=1$, Laughlin's variational wave function, obtained in the context of an electron gas in two dimensions interacting through the Coulomb $e^{2} / r$ potential, actually describes the noninteracting limit. This is consistent with the fact that the electronic charge $e$ in these solutions appears only in a product with the magnetic field, as $e B_{0}$, reflecting just the magnetic force on the electron.

\section{References}

[1] Landau, L. D., 1930, Z. Phys., 64, 629.

[2] Chakraborty, T., and Pietiläinen, P., 1995, The Quantum Hall Effects (Berlin: Springer).

[3] Von Klitzing, K., Dorda, G., and Pepper, M., 1980, Phys. Rev. Lett., 45, 494.

[4] Tsui, D. C., Störmer, H. L., and Gossard, A. C., 1982, Phys. Rev. Lett., 48, 1559.

[5] Laughlin, R. B., 1983, Phys. Rev. Lett., 50, 1395.

[6] Caillol, J. M., Levesque, D., Weis, J. J., and Hansen, J. P., 1982, J. stat. Phys., 28, 325.

[7] Levesque, D., Weis, J. J., and MacDonald, A. H., 1984, Phys. Rev. B, 30, 1056.

[8] Jancovici, B., 1981, Phys. Rev. Lett., 46, 386.

[9] Gradshteyn, I. S., and Ryzhiк, I. M., 1980, Table of Integrals, Series, and Products (New York: Academic Press). Integral 7.374.10 and the orthogonality of the Hermite polynomials yield this result.

[10] Lado, F., 1967, J. chem. Phys., 47, 5369.

[11] Ciftua, O., and Fantoni, S., 1997, Phys. Rev. B, 56, 13290.

[12] Kamilla, R. K., Jain, J. K., and Girvin, S. M., 1997, Phys. Rev. B, 56, 12411. 